\title{
Commentary on Christopher White, "Relationships between tonal stability and metrical accent in monophonic contexts."
}

\author{
DAVID TEMPERLEY \\ Eastman School of Music
}

\begin{abstract}
The following is a commentary of Christopher White's article on tonal stability and metrical accents.
\end{abstract}

Submitted 2017 June 3; accepted 2017 June 24.

KEYWORDS: meter, tonality, corpus, commentary

THIS study presents an interesting and valuable series of perceptual experiments on the relationship between tonality and meter. As the author observes, the relationship between tonal and metrical structure has been studied in various ways over the years. However, little attention has been given to what seems like the most basic question: does the metric stability of an event affect its perceived tonal stability, and/or vice versa? The current study seeks to answer these questions in four experiments. In the first three experiments, metrically ambiguous patterns were presented in a tonal context, to see if the tonally stable events were perceived to be metrically stronger (as measured by tapping responses); in all three studies, the answer was negative. (Here I use "tonal context" in a very general sense, simply to mean a context in which some pitch-classes are established as more stable or normative than others.) In the fourth study, a metrically accented but tonally ambiguous pattern was presented, to see if the metrically strong notes were perceived as more tonally stable (as measured by subjects' choice of harmony to accompany the pattern). Such an effect was found, though metrical strength seemed to affect the status of the notes in relation to the harmony (as chord-tones versus non-chord-tones), rather than in relation to the key framework; I will return to this point.

While this work is admirable in many ways, I do see some possible areas for improvement, and further directions that the author (or other authors) might wish to pursue in the future. With regard to methodology, I find it puzzling that, in the first three experiments at least, only one data point was gathered from each subject. In Experiment 1, for example, each subject heard just a single pitch pattern (out of four possible patterns) in one tonal context. Surely it would have been possible, and desirable, to have each subject hear all four pitch patterns - hardly an onerous workload, even if the compensation was only a "snack"!

Still focusing on the first three experiments, one might ask: Would we expect tonally stable events to be heard as metrically strong? This leads to another question: Are tonal and metrical stability in fact correlated in musical practice? I am not aware of claims along these lines in music theory-for example, claims that tonic-triad scale-degrees tend to be placed on metrically strong beats.[1] Some corpus evidence is available in a study by Prince \& Schmuckler (2014), which White cites but does not discuss in any depth. Using a corpus of piano works by Bach, Mozart, Beethoven, and Chopin, Prince \& Schmuckler examined the distribution of scale-degrees on beats of different metrical strength. They present the number of events of each scale-degree at different metrical positions, considering only major-key pieces in 4/4 time (about 60,000 notes); I have reproduced this data here in Table 1. (A limitation of this data is that it assumes that each piece is entirely in its main key, not recognizing modulations.) Metrical positions are grouped into six categories, representing different metrical levels (listed here in descending order of metrical strength): beat 1 , beat 3 , beats 2 and 4 , weak 8th-note beats, weak 16th-note beats, and other beats (e.g. weak 32nd-note beats). I have further analyzed this data in several ways, shown at the bottom of Table 1. First, taking Krumhansl \& Kessler's experimentally-derived major-key tone-profile (1982) as the definition of tonal stability (as White and many other authors have done), we can examine the average tonal stability of all events in each metrical category. Beat 1 has the highest average tonal stability, and the "other" category has the lowest, but the differences are small, and tonal stability does not decrease monotonically as metrical strength decreases; the stability of beats 2 and 4 is very slightly lower than that of 8th- and 16th-note beats. Other metrics show a similar pattern. The entropy of each distribution basically indicates how even it is; a more even (uniform) distribution results in higher entropy. If tonal stability correlates with metrical strength, 
we might expect distinctions between scale-degrees to be more marked at higher metrical levels, yielding lower entropy. Indeed, entropy is lowest for the downbeats and highest for "other," but the differences are again small. A third metric simply counts the number of diatonic (within-scale) notes as a proportion of the total, and a fourth metric counts the number of tonic-triad notes as a proportion of the diatonic ones; these two metrics show similar patterns to the first two. Perhaps the most striking pattern is found in the "tonictriad / diatonic" measure; in this case, the proportion of tonic-triad notes is somewhat higher on downbeats and lower on "other" beats. I will return to this point.

Table 1. Distribution of pitch-classes across metrical positions of a $4 / 4$ measure in a corpus of major-key classical pieces, transposed to C major. Rows C through B (except the rightmost column) are from Prince \& Schmuckler (2014); the following rows show my own analysis of this data. See the text for further explanation.

\begin{tabular}{|c|c|c|c|c|c|c|c|}
\hline & Beat 1 & Beat 3 & Beats 2,4 & 8ths & 16ths & Other & $\begin{array}{l}\text { Krumhansl- } \\
\text { Kessler major } \\
\text { profile (1982) }\end{array}$ \\
\hline $\mathrm{C}$ & 3356 & 2562 & 2154 & 1322 & 530 & 36 & 6.35 \\
\hline $\mathrm{Db}$ & 320 & 374 & 339 & 214 & 70 & 10 & 2.23 \\
\hline D & 1859 & 1978 & 1723 & 1123 & 481 & 31 & 3.48 \\
\hline $\mathrm{Eb}$ & 401 & 359 & 334 & 176 & 75 & 12 & 2.33 \\
\hline E & 2149 & 1877 & 1650 & 1140 & 423 & 30 & 4.38 \\
\hline F & 1588 & 1475 & 1358 & 813 & 308 & 22 & 4.09 \\
\hline F\# & 531 & 601 & 614 & 384 & 149 & 13 & 2.52 \\
\hline G & 3365 & 2681 & 2161 & 1528 & 612 & 41 & 5.19 \\
\hline $\mathrm{Ab}$ & 517 & 500 & 451 & 254 & 77 & 13 & 2.39 \\
\hline A & 1586 & 1474 & 1248 & 895 & 344 & 26 & 3.66 \\
\hline $\mathrm{Bb}$ & 307 & 376 & 319 & 215 & 80 & 11 & 2.29 \\
\hline B & 1598 & 1468 & 1302 & 819 & 356 & 26 & 2.88 \\
\hline Total & 17577 & 15725 & 13653 & 8883 & 3505 & 271 & \\
\hline $\begin{array}{l}\text { Avg KK } \\
\text { stability }\end{array}$ & 4.351 & 4.209 & 4.166 & 4.181 & 4.19 & 3.985 & \\
\hline Entropy & 3.195 & 3.277 & 3.304 & 3.278 & 3.25 & 3.428 & \\
\hline Dia/all & 0.882 & 0.860 & 0.849 & 0.860 & 0.871 & 0.782 & \\
\hline $\begin{array}{l}\text { TTtriad/ } \\
\text { dia }\end{array}$ & 0.572 & 0.453 & 0.514 & 0.522 & 0.512 & 0.395 & \\
\hline
\end{tabular}

Altogether, this corpus data suggests that tonal stability and metrical stability are only weakly correlated.[2] It is not clear that listeners would even pick up on such a weak association. In light of this, it is perhaps not surprising that listeners showed no tendency to hear tonally stable notes as metrically strong. We should note, also, that Prince \& Schmuckler's corpus data is from classical music; classical music probably represents part of the musical experience of White's subjects (undergraduate music students), but not all of it or (perhaps) even most of it. It would be interesting to look at similar data from popular music, 
though complications arise here, notably syncopation; many melodic notes in rock are displaced from the strong beats that they "belong" on (Temperley, 1999).

White's fourth experiment is initially presented as "flipping the paradigm"- examining the effect of meter on tonal stability, rather than vice versa. But as the author acknowledges, the experiment has a rather serious confound. Subjects heard a pattern of two alternating notes (e.g. C-D) with one note or the other accented, and decided which of three dominant seventh chords (F7, G7, or Bb7) provided the best fit to the pattern. What pattern of results would we expect here? As the author notes, each of the three chords specifies a unique key (or rather a unique tonic - a pair of parallel major and minor keys): $\mathrm{Bb}, \mathrm{C}$, and Eb, respectively. If listeners expect the metrically accented note to be more stable within the key, then they might infer a key of $\mathrm{Bb}$ if $\mathrm{D}$ was accented (since $\mathrm{D}$ is within the tonic triad of that key), hence favoring $\mathrm{F} 7$, and a key of $\mathrm{C}$ if $\mathrm{C}$ was accented, hence favoring G7. Let us call this the "tonal prediction." But an alternative prediction is that listeners might favor a chord containing the metrically accented note - on the grounds that metrically accented notes are more likely to be chord-tones. By this "harmonic prediction," listeners should favor F7 when $\mathrm{C}$ is accented (since F7 contains $\mathrm{C}$ ) and $\mathrm{G} 7$ or Bb7 when D is accented. While I am not aware of statistical evidence on the metrical placement of chord-tones and non-chord-tones, it is, I think, conventional wisdom that non-chord-tones such as passing tones and neighbor tones are most often (though certainly not always) metrically weak; e.g., "passing tones normally occur on unaccented beats or parts of the beat" (Aldwell \& Schachter, 2003, 334). In developing the harmonic analysis model presented in Temperley (2001), I found that it improved performance to include a preference for non-chord-tones to be metrically weak. By contrast, there is little theoretical support for the "tonal prediction," as noted above.

The evidence from White's study strongly supports the harmonic prediction over the tonal one. F7 was chosen much more often when $\mathrm{C}$ was accented rather than $\mathrm{D}$; for $\mathrm{G} 7$, the reverse pattern occurred. (Oddly, $\mathrm{Bb} 7$ was chosen almost equally often in the two cases.) Thus, if there was any tendency to hear the metrically accented note as tonally stable in relation to the key, it was dominated by the stronger preference to hear it as being part of the chord. I wonder if there might be a way of excluding the harmonic factor and testing the tonal prediction more directly. Perhaps one could play the melodic pattern unaccompanied, with one note or the other accented, followed by a cadence in one key or another, and ask the listener which cadence provided the better fit; this, it seems to me, would truly be "flipping the paradigm," more so than what the author actually did.

While White acknowledges the harmonic confound mentioned above, it seems to get a bit lost in his discussion of the experiment. He writes: "the overall data [from Experiment 4] suggest connections between metrical hierarchies and preferences for tonally-orienting harmonizations" (14); the experiment shows, he says, that "metrical hierarchies can affect tonal hierarchies." (14) But this seems to confuse tonal hierarchy (which he identifies elsewhere in the paper with distinctions in stability due to the key framework) with harmonic structure (distinctions in stability due to the local chord). White's study (specifically experiment 4) shows us only that metrical strength is correlated with the latter, not the former.

This leads me to my main point. As noted earlier, conventional music-theoretical wisdom suggests that chord-tones tend to be metrically strong; and White's experiment 4 suggests that listeners are sensitive to this. It seems safe to assume, also, that tonic harmony is more frequent than other chords. In a small corpus of classical excerpts (Temperley, 2009), I found that tonic harmony was present $40.6 \%$ of the time, far more than any other harmony. If tonic harmony is the most frequent harmony, and chord-tones tend to be metrically stronger than non-chord-tones, then that alone might cause tonic-triad scale-degrees to occur on strong beats slightly more than other scale-degrees; and such a "slight" difference is exactly what we observe in Prince \& Schmuckler's data. In short, all the evidence - theoretical wisdom, corpus data, and White's experimentssuggests that there is a connection between meter and "tonal stability" broadly defined; but it is a connection that relates primarily to harmony, rather than to key.

A final, somewhat parenthetical, point: One might wonder if there is a correlation between metrical structure and harmonic structure itself. That is, does tonic harmony tend to occur (i.e. start) on strong beats more often than other harmonies do? Temperley and De Clercq (2013) found such an effect in rock (using the Rolling Stone corpus, a set of 200 harmonic analyses of rock songs), and I suspect it is true in classical music as well. If so, this might be another factor giving rise to a correlation between metrical stability and tonal stability at the note level (if we assume that most notes within the span of a harmony are part of the harmony). This seems most relevant to higher metrical levels, since harmonies rarely change at lower levels. (In the Rolling Stone corpus, only about 5\% of chord changes occur below the half-note level.) Also, it would only affect notes that occur right at the beginning of the harmony. (For example, if I tends to occur more on beat 1 , and $\mathrm{V}$ on beat 3 , this might cause beat 2 to have more tonic-triad notes than beat 4 , but this would not 
give rise to a correlation with between tonal stability and metrical strength, since beats 2 and 4 are metrically equivalent.) Thus, I would expect it to have only a very small effect on the overall correlation between metrical stability and tonal stability at the note level.

\section{NOTES}

[1] As observed by White, several of Lerdahl \& Jackendoff's (1983) preference rules pertain to the interaction between meter and tonal structure. These rules relate to Lerdahl \& Jackendoff's hierarchical view of pitch structure, and are rather difficult to translate into more conventional terms. In general, I would say they express a preference for changes of harmony on strong beats, and also perhaps a preference for tonic harmony on strong beats. I will return to both of these points below.

[2] Prince \& Schmuckler actually compute the correlation between tonal stability and metrical stability in a more precise way. Using Krumhansl \& Kessler's (1982) tone-profiles, and "beat-profiles" (representing the perceived stability of different metrical positions) created by Palmer \& Krumhansl (1990), they compute the correlation between the average metrical stability of scale-degrees (in the corpus) and their tone-profile stability, as well as the correlation between the average tonal stability of each metrical position (in the corpus) and its beat-profile stability; in all cases, the correlations were strongly positive. To my mind, however, the metrics I show in Table 1 provide more intuitive ways of judging the strength of the relationship.

\section{REFERENCES}

Aldwell, E., \& Schachter, C. (2003). Harmony and Voice Leading (3rd ed.). Belmont, CA: Thomson/Schirmer.

Krumhansl, C., \& Kessler, E. (1982). Tracing the dynamic changes in perceived tonal organization in a spatial representation of musical keys. Psychological Review, 89, 334-368. https://doi.org/10.1037/0033295X.89.4.334

Lerdahl, F., \& Jackendoff, R. (1983). A Generative Theory of Tonal Music. Cambridge, MA: The MIT Press.

Palmer, C., \& Krumhansl, C. (1990). Mental representations for musical meter. Journal of Experimental Psychology: Human Perception and Performance, 16, 728-741. https://doi.org/10.1037/0096-1523.16.4.728

Prince, J., \& Schmuckler, M. (2014). The tonal-metric hierarchy: A corpus analysis. Music Perception, 31, 254-270. https://doi.org/10.1525/mp.2014.31.3.254

Temperley, D. (1999). Syncopation in rock: a perceptual perspective. Popular Music, 18, 19-40. https://doi.org/10.1017/S0261143000008710

Temperley, D. (2001). The Cognition of Basic Musical Structures. Cambridge, MA: MIT Press.

Temperley, D. (2009). A statistical analysis of tonal harmony. <http://davidtemperley.com/kp-stats/>

Temperley, D., \& de Clercq, T. (2013). Statistical analysis of harmony and melody in rock music. Journal of New Music Research, 42, 187-204. https://doi.org/10.1080/09298215.2013.788039 